Spi $\mathrm{n}$ - $\mathrm{gl}$ ass and novel magnet i $\mathrm{c}$ behavi or in the spi nel - type Cu1- xAgxCr SnS4

\begin{tabular}{|l|l|}
\hline 著者 & $\begin{array}{l}\text { I SH KAMA Takahi ro, EBI SU Shuj i, NAGATA } \\
\text { Shoi chi }\end{array}$ \\
\hline $\begin{array}{l}\text { j our nal or } \\
\text { publ i cat i on ti tl e }\end{array}$ & Physi ca. B, Condensed mat ter \\
\hline vol une & 405 \\
\hline nunber & 7 \\
\hline page r ange & $1881-1889$ \\
\hline year & $2010-04$ \\
\hline URL & ht t p: //hdl . handl e. net /10258/488 \\
\hline
\end{tabular}


Spi $\mathrm{n}$ - $\mathrm{gl}$ ass and novel magnet i $\mathrm{c}$ behavi or in the spi nel - type Cu1- xAgxCr SnS4

\begin{tabular}{|l|l|}
\hline 著者 & $\begin{array}{l}\text { I SH KAMA Takahi ro, EBI SU Shuj i, NAGATA } \\
\text { Shoi chi }\end{array}$ \\
\hline $\begin{array}{l}\text { j our nal or } \\
\text { publ i cat i on ti tl e }\end{array}$ & Physi ca. B, Condensed mat ter \\
\hline vol une & 405 \\
\hline nunber & 7 \\
\hline page r ange & $1881-1889$ \\
\hline year & $2010-04$ \\
\hline URL & ht t p: //hdl . handl e. net /10258/488 \\
\hline
\end{tabular}




\title{
Spin-glass and novel magnetic behavior in the spinel-type $\mathrm{Cu}_{1-x} \mathrm{Ag}_{x} \mathrm{CrSnS}_{4}$
}

Takahiro Ishikawa, Shuji Ebisu,

Shoichi Nagata

Department of Materials Science and Engineering, Muroran Institute of Technology, 27-1 Mizumoto-cho, Muroran, Hokkaido, 050-8585 Japan

\begin{abstract}
A dual non-magnetic substitution system on $A$ - and $B$-sites in the spinel structure has been studied. The mother compound is a ferromagnet $\mathrm{CuCr}_{2} \mathrm{~S}_{4}$ with the Curie temperature $T_{\mathrm{c}} \simeq 380 \mathrm{~K}$. A system of $\mathrm{Cu}_{1-x} \mathrm{Ag}_{x} \mathrm{CrSnS}_{4}$, which is the same notation as $\left(\mathrm{Cu}_{1-x} \mathrm{Ag}_{x}\right)\left(\mathrm{Cr}_{0.50} \mathrm{Sn}_{0.50}\right)_{2} \mathrm{~S}_{4}$, has been prepared over the entire range of $0.00 \leq x \leq 1.00$ although the $\mathrm{Cr}-\mathrm{Sn}$ sublattice is unchanged in the fixed composition of 0.50 on $B$-sites. All these compounds exhibit the spin-glass phase with the freezing temperature $T_{\mathrm{g}}$ approximately at $16 \mathrm{~K}$ in $100 \mathrm{Oe}$. Since only $\mathrm{Cr}$ ions have the magnetic moment on the $B$ sites, the substitution of $\mathrm{Ag}$ for $\mathrm{Cu}$ on the $A$-sites does not influence strongly the spin-glass freezing behavior over the whole composition range. Nevertheless, the magnetization of $\mathrm{Cu}_{1-x} \mathrm{Ag}_{x} \mathrm{CrSnS}_{4}$ with $x=0.50$ and 0.55 causes a broad upturn hump over 30 to $130 \mathrm{~K}$ where the spin-glass phase is broken. Strong magnetic field dependence of this hump anomaly has been observed with an irreversibility between zero-field-cooled ( $\mathrm{ZFC}$ ) and field-cooled (FC) magnetizations even though above $T_{\mathrm{g}}$. The hump is suppressed in higher fields and collapsed down at approximately $1.0 \mathrm{kOe}$ with a tiny trace quantity of the anomaly where the difference between the ZFC and $\mathrm{FC}$ processes disappears. The specimen with $x=0.45$ shows a small hump anomaly in low field less than 20 Oe which corresponds to a precursor of the huge anomaly for $x=0.50$. The hump anomaly could be attributed to a formation of the cluster-glass. The spin-clusters are embedded in the matrix of spin-glass el-
\end{abstract}

Email addresses: naga-sho@mmm.muroran-it.ac.jp (Shoichi Nagata) 
ements in high degree of disorder without long-range order. All the spins eventually are frozen below $T_{\mathrm{g}}$. The strange magnetic freezing originates from the delicate dual substitutions. The mechanism of the anomaly is far from a complete picture and remains enigmatic.

Key words: Spinel-sulfide $\mathrm{Cu}_{1-x} \mathrm{Ag}_{x} \mathrm{CrSnS}_{4}$, Two-sites substitution effect, Spin-glass, Cluster-glass, Magnetic hysteresis loops

\section{Introduction}

Thiospinels exhibit a wide variety of physical properties. One of these spinels, $\mathrm{CuCr}_{2} \mathrm{~S}_{4}$ is a compound of great interest as it shows ferromagnetism. $\mathrm{CuCr}_{2} \mathrm{~S}_{4}$ is a metallic ferromagnet with a Curie temperature $T_{\mathrm{c}} \simeq 380 \mathrm{~K}$. Versatile features of this typical ferromagnet have been investigated as a mother compound for the substitution effects. The formula unit has a net magnetic moment close to $5.0 \mu_{\mathrm{B}}$. It has been established that $\mathrm{CuCr}_{2} \mathrm{~S}_{4}$ has the mixed valence as $\mathrm{Cu}^{+} \mathrm{Cr}^{3+} \mathrm{Cr}^{4+} \mathrm{S}_{4}{ }^{2-}$, here $\mathrm{Cr}^{3+}$ ion with $3 \mu_{\mathrm{B}}$ and $\mathrm{Cr}^{4+}$ ion with $2 \mu_{\mathrm{B}}$, and a $\mathrm{Cu}^{+}$ion has the closed shell. The $\mathrm{Cr}$ ions align parallel each other, as a result the formula unit of $\mathrm{CuCr}_{2} \mathrm{~S}_{4}$ has a net magnetic moment of $5 \mu_{\mathrm{B}}(1 ; 2 ; 3 ; 4 ; 5)$.

The chemically modified mixed valence spinel on $B$-sites, $\mathrm{CuCrZrS}_{4},(6 ; 7$ : $8 ; 9 ; 10 ; 11$ ) has provided a way to spin-glass phase. A common characteristic of the spinel families $\mathrm{Cu}\left(\mathrm{Cr}_{1-x} \mathrm{M}_{x}\right)_{2} \mathrm{~S}_{4}$ (where $\mathrm{M}=\mathrm{Ti}, \mathrm{Zr}$, Hf) is that the substitution of $\mathrm{M}$ element for $\mathrm{Cr}$ atom proceeds from ferromagnetic to spinglass behavior $(12 ; 13)$. It would be a natural extension and expectation that $4+$ valence ion of $\mathrm{Sn}^{4+}$ is a candidate of the substitution for $\mathrm{Cr}$ on the $B$-sites. The quaternary spinel $\mathrm{CuCrSnS}_{4}$ has been studied extensively $(14 ; 15 ; 16 ; 17 ; 18 ; 19 ; 20 ; 21)$.

A spinel-type $\mathrm{AgCr}_{2} \mathrm{~S}_{4}$ has never been synthesized so far, because of the difficulty of synthesis. The occupation of $\mathrm{Ag}$ atom on the $A$-sites in the spinel compound is rarely seen. A few spinel compounds exist with occupying $\mathrm{Ag}$ atom on the $A$-sites $(22 ; 23)$. The $\mathrm{Ag}$ based spinels $\mathrm{Ag}\left(\mathrm{Cr}_{1-x} \mathrm{Sn}_{x}\right)_{2} \mathrm{~S}_{4}(x=$ $0.50,0.60$, and 0.70 ) have been successfully prepared recently and the results of the spin-glass characteristics have been reported (24).

We have focused the present study on the non-magnetic Ag substitution for $\mathrm{Cu}$ on the $A$-sites although the $\mathrm{Cr}$-Sn sublattice is unchanged in the fixed composition for $B$-sites. Here $50 \% \mathrm{Cr}$ and $50 \% \mathrm{Sn}$ atoms are dis- 
tributed randomly on the octahedral $B$-sites (25). Single-phase specimens with the dual substitution were obtained after optimization of the sintering conditions. This system of $\mathrm{Cu}_{1-x} \mathrm{Ag}_{x} \mathrm{CrSnS}_{4}$, which indicates the same notation as $\left(\mathrm{Cu}_{1-x} \mathrm{Ag}_{x}\right)\left(\mathrm{Cr}_{0.50} \mathrm{Sn}_{0.50}\right)_{2} \mathrm{~S}_{4}$, can be prepared over the entire range of $0.00 \leq x \leq 1.00$.

The first stage of experiments is to find how strongly or weakly the spinglass phase is influenced by non-magnetic Ag substitution. This expectation has become true because the spin-glass behavior does not vary over the whole composition range with the freezing temperature $T_{\mathrm{g}}$ approximately at $16 \mathrm{~K}$ in 100 Oe. Since only $\mathrm{Cr}$ ions have the magnetic moment on the $B$-sites, the substitution of $\mathrm{Ag}$ for $\mathrm{Cu}$ on the $A$-sites does not strongly influence the magnetic properties.

Nevertheless, we have discovered a strange upturn hump anomaly in the temperature variation of magnetization $M$ for the compositions of $x=0.50$ and 0.55 . This hump anomaly arises in the temperature region between 30 and $130 \mathrm{~K}$, above the freezing temperature $T_{\mathrm{g}}$. This upturn hump is seen in rather low field and eventually suppressed in higher field than approximately $1.0 \mathrm{k}$ Oe. The temperature and field dependences of this hump anomaly of $M$ have been examined.

A possibility of formation of the cluster-glass in the matrix of spin-glass elements will be proposed, because an irreversibility effect was detected for the field application. The isothermal magnetic hysteresis loops were measured for $x=0.50$ at several temperatures. The results of these $M-H$ curves indicate also specific features. The present study is mainly concerned with the experimental finding in the dc magnetization measurements for $x$ $=0.50$.

\section{Experimental Methods}

The initial study of sample preparations is reported by Hasegawa (24). The highest purity specimens were produced by different heat treatments as shown in Fig. 1. The precise sintering temperature has to be adapted for each composition. This empirical method can lead to the optimum condition for synthesis of the high purity specimens of $\mathrm{Cu}_{1-x} \mathrm{Ag}_{x} \mathrm{CrSnS}_{4}$. These specimens were heated to the specific temperature as indicated in Fig. 1 and kept at these temperatures for 4 days, then were annealed at $673 \mathrm{~K}$ for 4 hours. All the prepared specimens were found to be stable under air after the synthesis. The identification of crystal structure and the determination of 


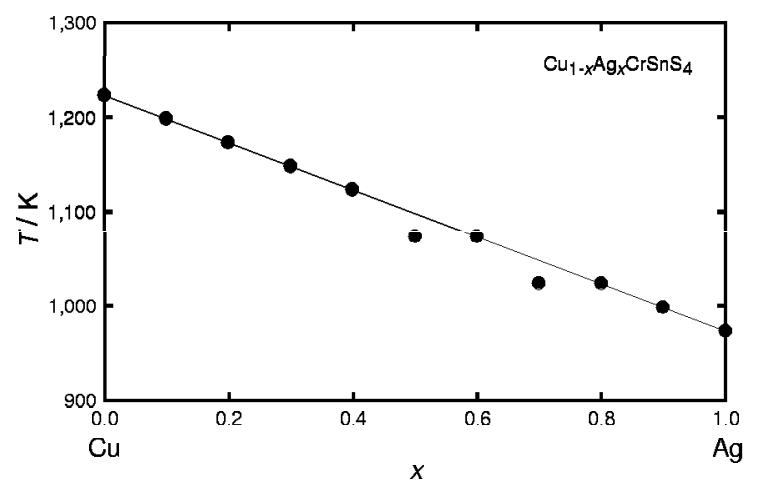

Figure 1: Optimum condition of the heat treatment for the sample preparation of $\mathrm{Cu}_{1-x} \mathrm{Ag}_{x} \mathrm{CrSnS}_{4}$. 
lattice constants were carried out by X-ray powder diffraction (XRD) using $\mathrm{Cu} \mathrm{K} \alpha$ radiation at room temperature. The XRD data were analyzed by the Rietveld simulation method with RIETAN-2000 (25).

The dc magnetization measurements of powder specimens were performed with a quantum design superconducting quantum interference device (rfSQUID) magnetometer over the temperature range over 2.0-350 K. For many specimens, the experimental results showed the appearance the irreversible effect between the zero-field-cooled (ZFC, initial magnetization, after cooling the sample to the lowest temperature in zero field, a field was applied and the data were taken with increasing temperature) and the field-cooled (FC, application of field before cooling) magnetizations (26). The isothermal magnetic hysteresis curves were measured in the $\mathrm{ZFC}$ and $\mathrm{FC}$ processes at several temperatures. The demagnetizing field corrections have not been applied to any of our magnetic data because of the small magnitude of the magnetization.

\section{Results and Discussion}

\subsection{Lattice constant}

X-ray data have been analyzed in terms of a cubic spinel structure with the space group $F d \overline{3} m$ (No. 227). The X-ray diffraction (XRD) patterns at room temperature give evidence that all the $\mathrm{Cu}_{1-x} \mathrm{Ag}_{x} \mathrm{CrSnS}_{4}$ have the spinel type structure. Representative diffraction profiles are shown in Fig. 2. The Rietveld simulation method with RIETAN-2000 verifies the random distribution in the $A$ - and $B$-sites, respectively (25). The site preference is exactly examined and confirmed that $\mathrm{Cu}$ and $\mathrm{Ag}$ occupy at only A-sites and $\mathrm{Cr}$ and $\mathrm{Sn}$ atom occupy at $B$-sites. Occupation percentage and the randomness are verified. However, the Rietveld refinement to adjust the inter atomic distances has not been done in this substitution system.

More detailed discussion concerned with distribution will be mentioned in later subsection for the specific specimen with $x=0.50$. The lattice constant, $a$, obtained by the least square method, varies as shown in Fig. 3 at room temperature. Here, these values of $x$ are given by the initial stoichiometry of the reacting constituents. The lattice constant increases linearly with increasing Ag substitution $x$ and obeys Vegard's law. The unit sell size is $a=10.158 \mathrm{~A}$ for $\mathrm{CuCrSnS}_{4}$ in the present study and is reported to be $a=$ $10.440 \AA$ for $\mathrm{AgCrSnS}_{4}$, (24) respectively. 


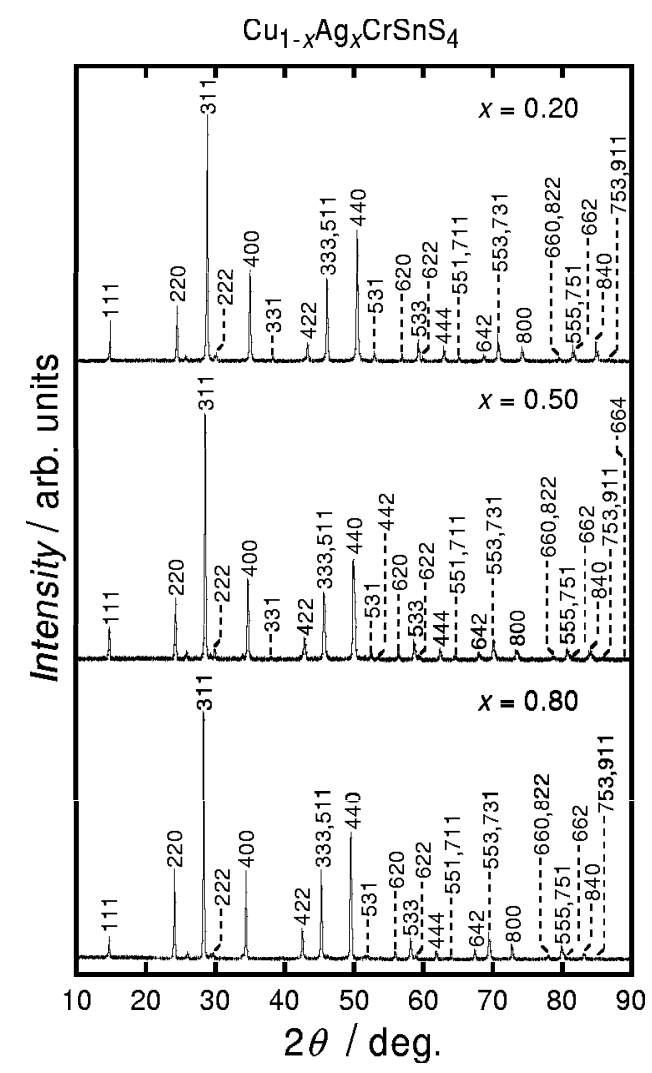

Figure 2: X-ray diffraction profiles of $\mathrm{Cu}_{1-x} \mathrm{Ag}_{x} \mathrm{CrSnS}_{4}$ for powdered specimens with $x=$ $0.20,0.50$, and 0.80 at room temperature. 


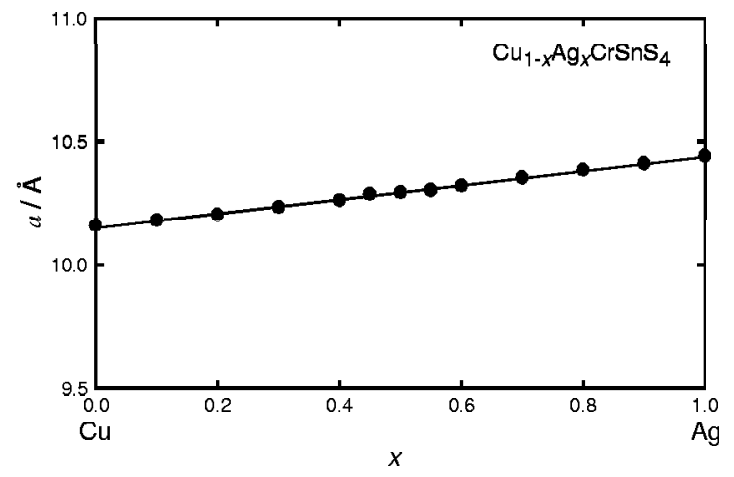

Figure 3: Lattice constant $a$ as a function of $\mathrm{Ag}$ composition $x$ at room temperature. 


\subsection{Temperature dependences of magnetization in a field of $100 \mathrm{Ot}$}

Figs. 4 and 5 display the temperature dependences of the magnetization for the specimens of $x=0.00$ to 1.00 in a constant field of 100 Oe. The magnetization as a function of temperature exhibits the spin-glass characteristics below $T_{\mathrm{g}}$. Their bifurcation from the $\mathrm{ZFC}$ and $\mathrm{FC}$ processes arises at approximately $16 \mathrm{~K}$ with decreasing temperature which is almost independent on the composition $x$ as shown in Figs. 4 and 5. The non-magnetic Ag substitution for $\mathrm{Cu}$ atom on $A$-sites has given a fairly weak modification of spin-glass feature, on the contrary the $B$-sites substitution influences strongly the spin-glass characteristics $(12 ; 24)$. Since the magnetic moments comes from $\mathrm{Cr}$ atom at $B$-sites, then, these results of Figs. 4 and 5 are reasonable to inderstand.

Since the sulfides such as $\mathrm{Cu}_{1-x} \mathrm{Ag}_{x} \mathrm{CrSnS}_{4}$ have a covalent bond nature concerning the direction, the magnetic interaction is influenced more strongly by the magnetic dilution between the nearest neighbor (n. n.) interactions than that of pure ionic bond as oxides. Furthermore, the ionic radius is $1.74 \AA$ for $\mathrm{S}^{2-}$ which is much larger than that of $1.32 \AA$ for $\mathrm{O}^{2-}$. The difference of radius between the metallic ions and $\mathrm{S}^{2-}$ ions becomes larger, so that the volume contraction is more distinct with decreasing temperature in comparison with oxides. The $\mathrm{Sn}$ substitution for $\mathrm{Cr}$ would introduce the complex interactions. The antiferromagnetic interaction would survive in the next nearest neighbor (n.n.n.) interactions. Consequently these interactions can lead the system to the frustrated situation with low asymptotic Weiss temperature.

\subsection{The (Yurie-Weiss law}

As a representative specimen, Fig. 6 stands for the temperature dependences of the magnetization $M$ and the inverse susceptibility $\chi^{-1}$ for $x=$ 0.70 , here, the susceptibility is referred to $\chi=M / H$. The deviations from the Curie-Weiss law, as illustrated in Fig. 6, come presumably from the influence of the isolated clusters of spin. The $\chi^{-1}$ for $x=0.40,0.50$, and 0.60 are given in Fig. 7. In high temperature region, $\chi$ fits the Curie-Weiss law. It is noticed that the specimen of $x=0.50$ exhibits an irregular behavior below $130 \mathrm{~K}$, while it is normal above $130 \mathrm{~K}$, which will be described in a next subsection.

The summary of the magnetic results of $\mathrm{Cu}_{1-x} \mathrm{Ag}_{x} \mathrm{CrSnS}_{4}$ is given in Table 1 . These numerical values are extracted from the Curie-Weiss law. The value of effective moment $p_{\text {eff }}$ Cr-atom ${ }^{-1}$ has been obtained. The numerical 




Figure 4: Temperature dependences of magnetization of $\mathrm{Cu}_{1-x} \mathrm{Ag}_{x} \mathrm{CrSnS}_{4}$ in a constant field of 100 Oe for $x=0.00,0.20$ and 0.40 . The differences between zero-field cooled (ZFC) and field-cooled (FC) magnetizations are indicated, which arises from the spinglass characteristics below $T_{\mathrm{g}}$. 


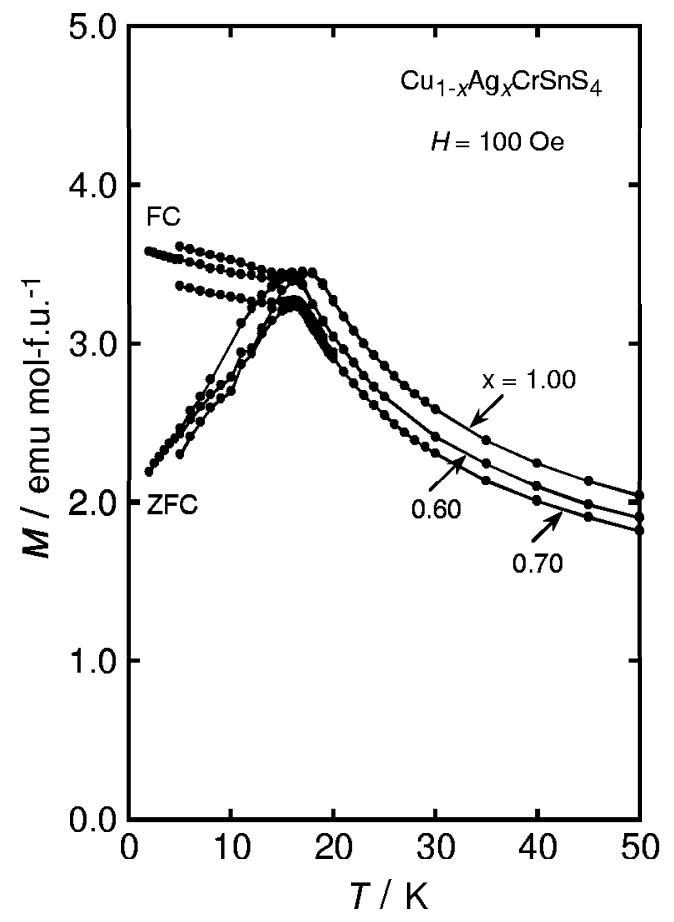

Figure 5: Temperature dependences of magnetization of $\mathrm{Cu}_{1-x} \mathrm{Ag}_{x} \mathrm{CrSnS}_{4}$ in a constant field of 100 Oe for $x=0.60,0.70$ and 1.00 . 


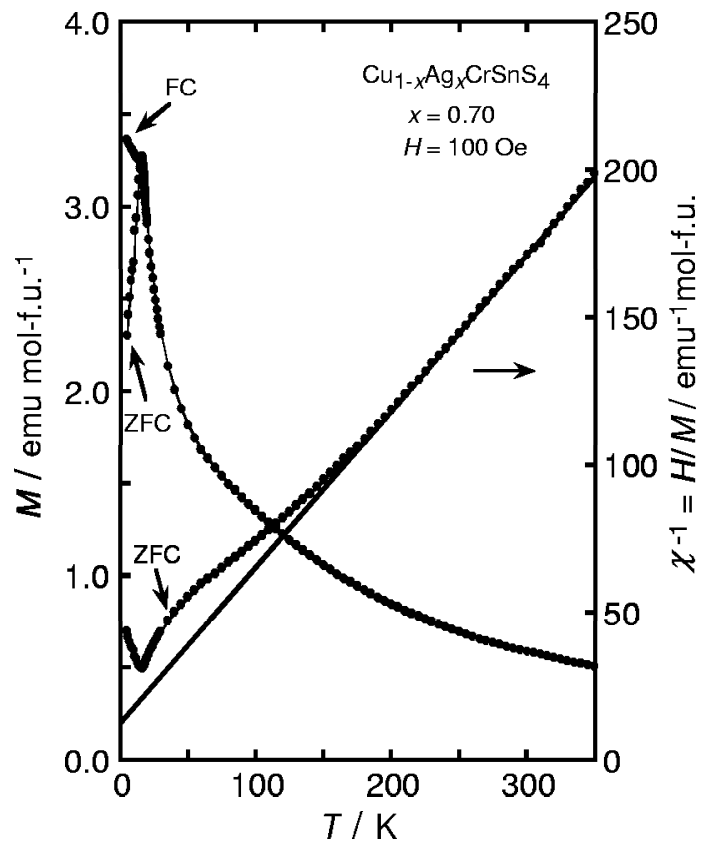

Figure 6: Magnetization $M$ and the inverse susceptibility $\chi^{-1}=(M / H)^{-1}$ as a function of temperature in a constant field of 100 Oe for $x=0.70$. 


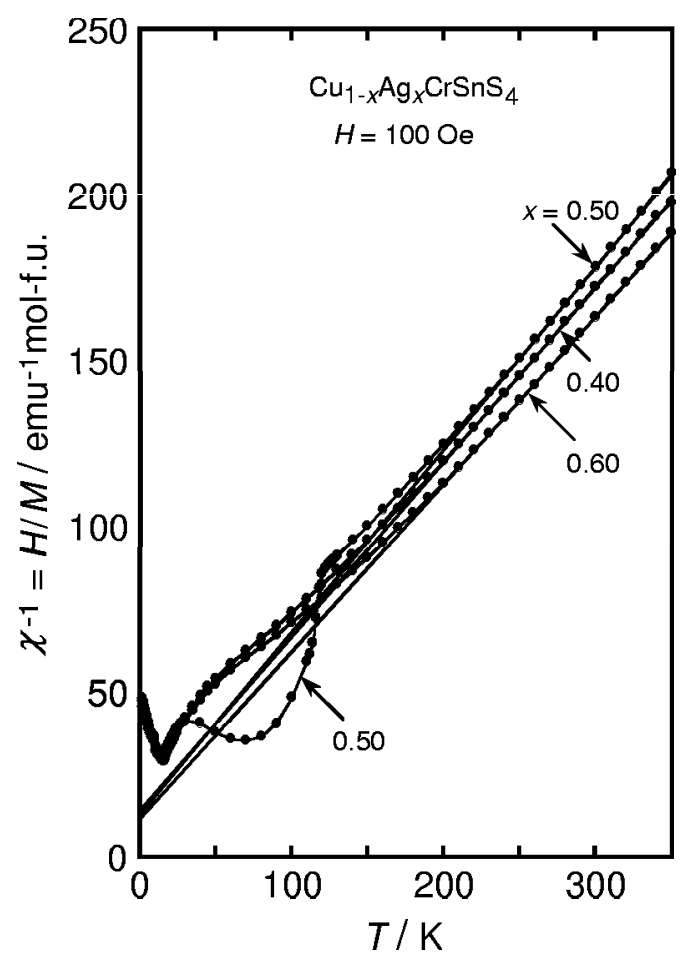

Figure 7: Temperature variations of inverse magnetic susceptibility $\chi^{-1}=(M / H)^{-1}$ for $\mathrm{Cu}_{1-x} \mathrm{Ag}_{x} \mathrm{CrSnS}_{4}$ with $x=0.40,0.50$, and 0.60. Deviation from the straight line is seen for $x=0.50$ below $130 \mathrm{~K}$, while it is normal above $130 \mathrm{~K}$. 
evaluation indicates intensely that $\mathrm{Cr}^{3+}$ ion has spin value of $S=3 / 2$. The asymptotic Weiss temperature $\theta$ has negative sign with dominant antiferromagnetic interaction. It verifies the introduction of the antiferromagnetic interaction through the substitution, reflecting the nature of competing interactions between ferro- and antiferromagnetic exchange interactions in the spin-glass formation at lower temperatures.

\subsection{Hump anomaly and the experimental reproducibility}

A novel behavior in $M$ is found for $\mathrm{Cu}_{0.55} \mathrm{Ag}_{0.45} \mathrm{CrSnS}_{4}$. Fig. 8. shows the field dependence of the magnetization. In the region of $T_{\mathrm{g}} \leq T \leq 110 \mathrm{~K}$, strange small humps of $M$ are seen in only low fields of 10 and 20 Oe, indicating the difference between the $\mathrm{ZFC}$ and $\mathrm{FC}$ processes. The result for 100 Oe shows a collapse of the hump, see inset of Fig. 8 These data send a signal the specimen of $x=0.50$ toward a huge anomaly. It should be noticed that, the other compositions, except $x=0.50$ and 0.55 , may exhibit the same results such as Fig. 8 in the restricted lower field. We have measured temperature variation of $M$ in only 100 Oe as shown in Figs. 4 and 5 .

Striking features in $M$ are found for $x=0.50$ and 0.55 in $\mathrm{Cu}_{1-x} \mathrm{Ag}_{x} \mathrm{CrSnS}_{4}$. Figs. 9 and 10 indicate the field dependence of the magnetization over 5.0 to 1000 Oe. Fig. 11 displays the susceptibility, defined as $\chi=(M / H)$, which demonstrate a nonlinearity of the hump-like anomaly as a function of the magnetic field. A tendency toward the saturation is seen in the higher field.

The reproducibility of the experimental results of Figs. 9-11 will be mentioned now. Examinations for the different batches, thermal cycles to 300 $\mathrm{K}$ in the application of external fields, and the stability with time not less than half year, have been carried out. The reproducibility after thermal cycle between 5 and $300 \mathrm{~K}$ is exactly verified, five times up and down, for the specified specimen in Figs. 9-11. This specimen, here, has experienced the application of the field up to 1000 Oe. We made an inspection of deterioration with age or reduction of the properties of these specimens for the same specimen in Figs. 9-11, after 180 days from the first run of measurements. The specimen had been maintained at room temperature after the first measurements. Subsequent second and third shot operations of the measurements have yielded the same experimental results within the experimental errors. We have measured three specimens provided from independent different batches for $\mathrm{Cu}_{0.50} \mathrm{Ag}_{0.50} \mathrm{CrSnS}_{4}$ which were prepared in the same heat treatment given in section 2. All the experimental data for these independent specimens have verified the almost same results as shown in Figs. 9-11 within 


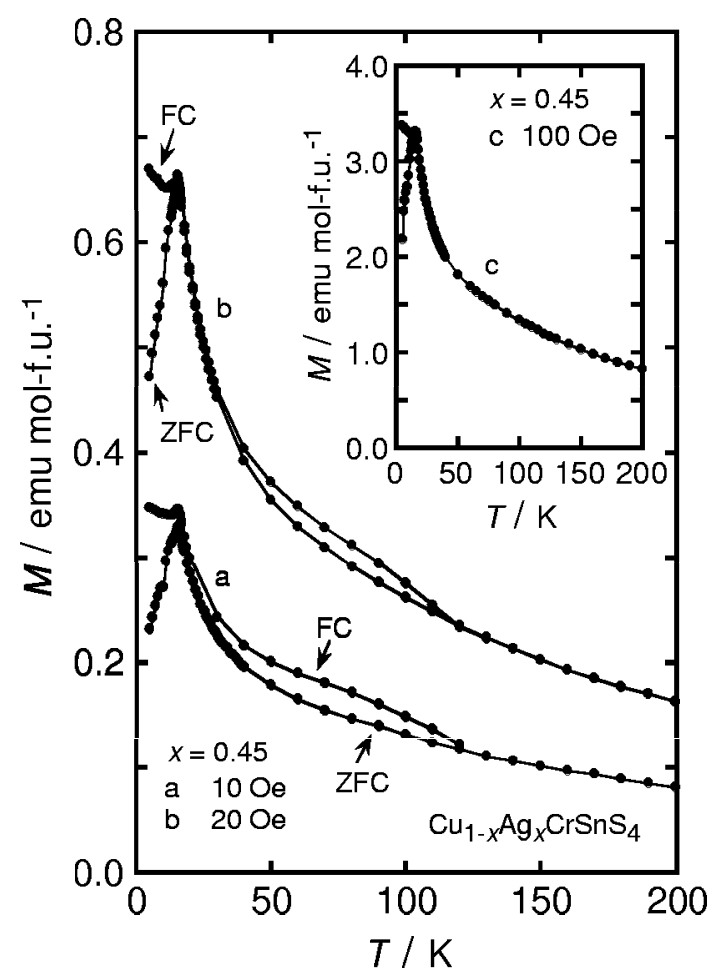

Figure 8: Temperature dependences of the magnetization for $\mathrm{Cu}_{0.55} \mathrm{Ag}_{0.45} \mathrm{CrSnS}_{4}$, which indicates a precursor of the huge anomaly for $x=0.50$, see Fig. 9. The differences between $\mathrm{ZFC}$ and $\mathrm{FC}$ magnetizations are observed even above $T_{\mathrm{g}}$. A germination of the hump anomaly is seen in 10 and 20 Oe above $T_{\mathrm{g}}$. The inset shows that the hump shrinks in 100 Oe, in which the difference between ZFC and FC magnetizations disappears. 


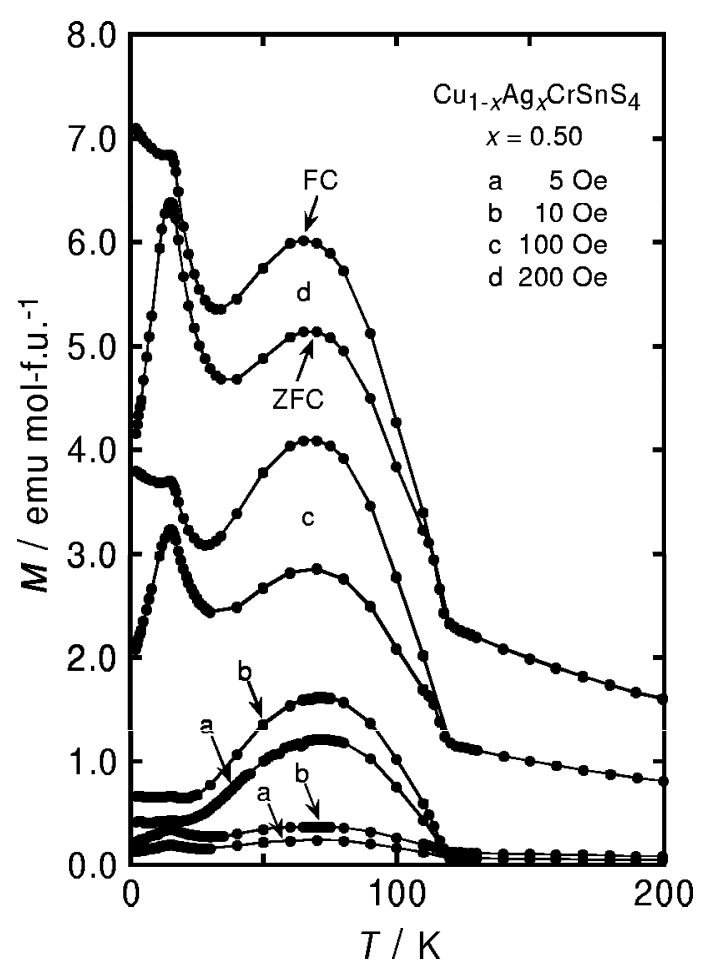

Figure 9: Variation of the magnetization with temperature in the fields from 5.0 to 200 Oe for $\mathrm{Cu}_{0.50} \mathrm{Ag}_{0.50} \mathrm{CrSnS}_{4}$. The differences between $\mathrm{ZFC}$ and $\mathrm{FC}$ magnetizations are observed even above $T_{\mathrm{g}}$. Low temperature spin-glass phase arises below $T_{\mathrm{g}} \simeq 16 \mathrm{~K}$. The broad hump anomaly above $T_{g}$, which is spread approximately over 30 to $130 \mathrm{~K}$, has been detected. This hump anomaly would be attributed to a formation of short-range spinclusters. The spin-glass and the spin-clusters develop in the different temperature regions, respectively. 


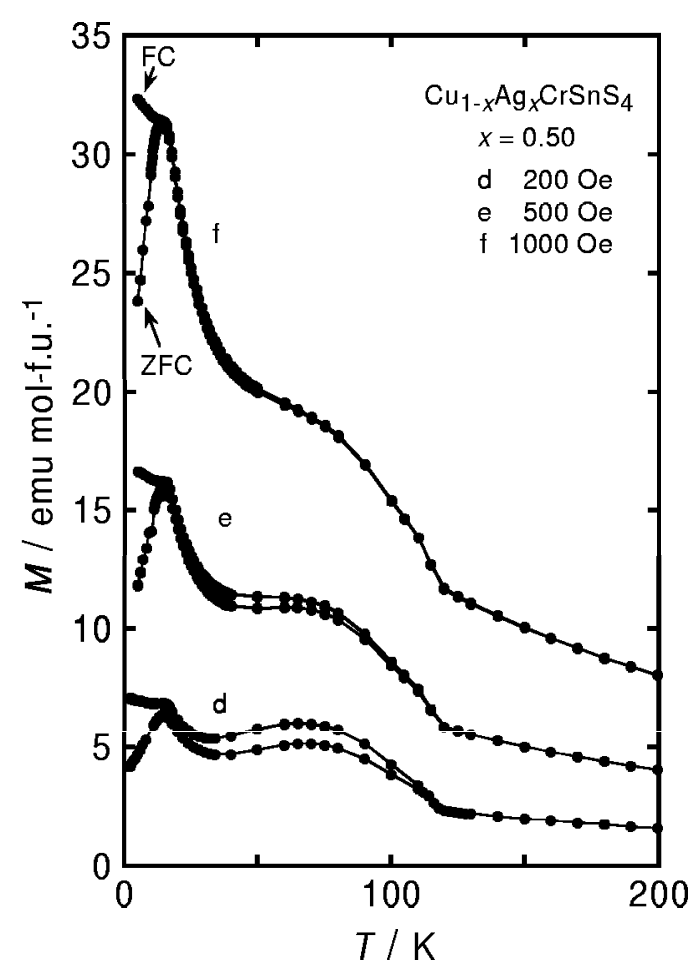

Figure 10: Temperature dependences of the magnetization in the fields of 200, 500, and 1000 Oe for $\mathrm{Cu}_{0.50} \mathrm{Ag}_{0.50} \mathrm{CrSnS}_{4}$. The hump anomaly seen in Fig. 9 has shrunk in a field of approximate $1000 \mathrm{Oe}$. The results for higher fields of 5000 and 10000 Oe give similar shape of the temperature variations of $M$ as seen in 1000 Oe, but these magnitudes of $M$ increase with the field. 


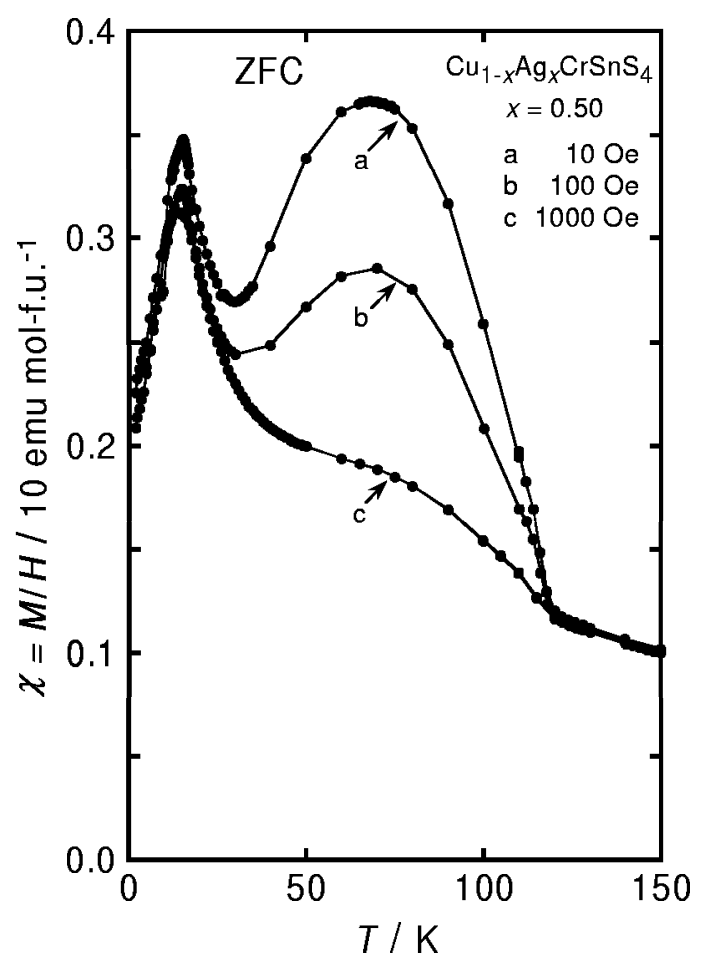

Figure 11: Non-linear behavior and the tendency toward a saturation of the magnetization. They are manifested as a function of the applied field in the region from 30 to $130 \mathrm{~K}$. These susceptibilities $\chi$, defined as $M / H$, are extracted from the experimental results of Fig. 9 and 10. The three fields of 10,100 , and 1000 Oe are shown and the only ZFC data are presented, for avoiding the overlap of drawing. 


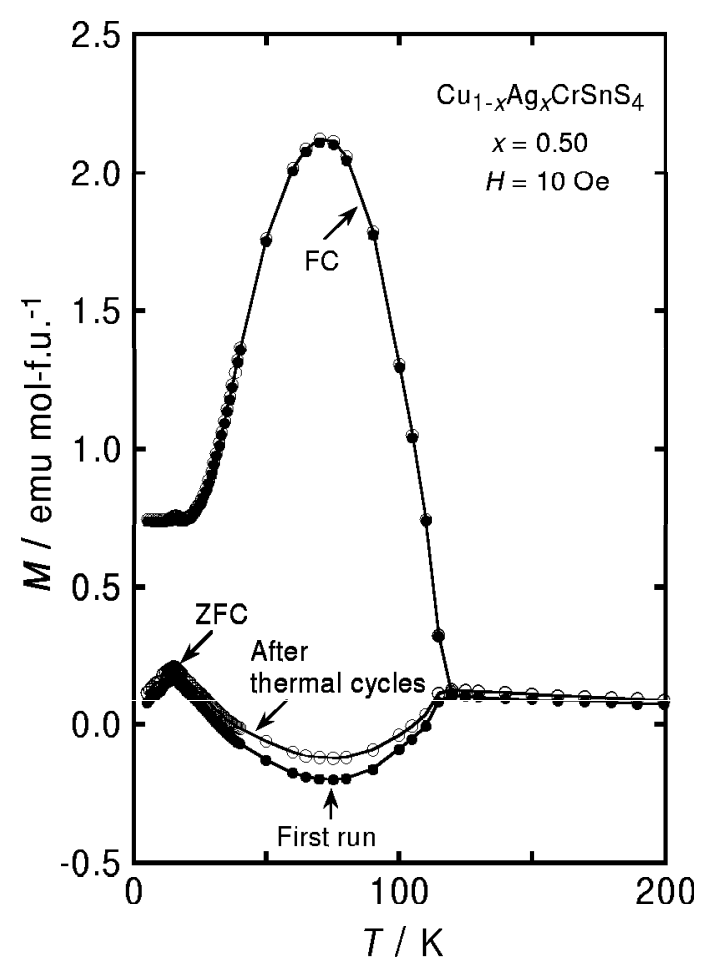

Figure 12: ZFC magnetization with negative value in 10 Oe between 32 and $120 \mathrm{~K}$ for $\mathrm{Cu}_{0.50} \mathrm{Ag}_{0.50} \mathrm{CrSnS}_{4}$. This negative value changes a little after the thermal cycles from the first observation. The specimen has experienced the several thermal cycles to $300 \mathrm{~K}$, and also the application of the field of 1000 Oe. The specimen is different from those of Figs. 9-11. This is chosen from different batch for the sample preparation. It is stressed that the magnetizations in higher field than 10 Oe exhibit the almost same results obtained in Figs. 9-10 up to 10000 Oe within the experimental errors. 
the experimental errors. It is stressed that the reproducibility of the results is excellent for three specimens. In addition, the specimens with $x=0.55$ and 0.58 have been also measured. The result of $x=0.55$ gives almost same data with $x=0.50$, while the results for $x=0.58$ provides intermediate magnitude between the $x=0.45$ and $x=0.50$, which is not shown to avoid confusion.

Fig. 12 demonstrates an exotic result in the ZFC process, for another specimen from the different batch. In rather low field of 10 Oe the negative value of magnetization is observed over the temperature region 32 to $120 \mathrm{~K}$ for one of the specimens with $x=0.50$, while the $\mathrm{FC}$ process has positive sign of the magnetization in the same temperature region. The absolute value of the magnetization in the negative region gets smaller approximately 20 percent than that of initial measurement, after the several thermal cycles to $300 \mathrm{~K}$ in the field up to 1000 Oe. It is noted that the residual magnetic field due to the set up in the apparatus is less than 10 Oe.

The crystal structure below $300 \mathrm{~K}$ has not been measured yet, but measured at only room temperature in the present study. A comment will be given now concerning the no-change of the structure. Fig. 10, for example, indicates a characteristic background curves in the temperature variation of $M$ for both of the $\mathrm{ZFC}$ and $\mathrm{FC}$ processes. Interpolation curves of $M$, from the two different regions $T<30 \mathrm{~K}$ and $T>130 \mathrm{~K}$ to the region of $30 K<T<130 \mathrm{~K}$, would merge well and connect smoothly in the field higher than 200 Oe, as seen in Fig. 10. Therefore, the crystal structure seems not to change below $130 \mathrm{~K}$ but be kept as same at the room temperature. If the structure should transform at certain temperature below $130 \mathrm{~K}$, we can expect a step-like change in the temperature variation $M$.

No structural transformation at low temperature, is reported by French group (17). They measured the X-ray diffraction down to $1.0 \mathrm{~K}$ for the similar spinel sulfide (but not the same compound), corresponding with $x \simeq 0.00$ (17), and then no structural transformation was detected. The present system of $\mathrm{Cu}_{1-x} \mathrm{Ag}_{x} \mathrm{CrSnS}_{4}$ shows the similar properties concerned with the spinglass behavior below $T_{\mathrm{g}}$, including the specimen with $x=0.50$. For $x=$ 0.50 , if the structure would change below $130 \mathrm{~K}$, we will expect absolutely different features for the spin-glass phase below $T_{\mathrm{g}}$ from them of Figs. 9-11. Even though the experimental facts mentioned above are indirect contact with the present sample, they are plausible to explain no structure change. As a consequence, the structure transformation will not take place at low temperature region for all the compositions. 


\subsection{Composition dependence of $X$-ray diffraction}

The strange magnetic hump in $M$ originates from the delicate influence from the dual non-magnetic substitution on the $A$ - and $B$-sites. These magnetic properties comes from, presumably, a little deviation from the complete random distribution on the $A$ - and $B$-sites. The Rietveld simulation can confirm that the random distribution means for only each site (25). The inhomogeneity, such as short-range composition fluctuation or magnetic clustering is poorly resolved by means of the ordinary X-ray powder diffraction. It is hard situation to detect any evidence of the deviation from the complete random configuration for the specimen for $x=0.50$. Nevertheless, we have made an attempt to analyze the X-ray peak height (peak intensity) on the basis of the Rietveld simulation. Fig. 13 stands for a variation of the peak intensity of the X-ray diffraction of the Miller indices of 440 as a representative diffraction. The index 440 leads to composition dependence, where a broad minimum around $x=0.50$ can be seen. The index 533 also gives the similar tendency as shown in Fig. 13. The reasonable explanation of the composition dependence of Fig. 13 is less clear at present. The composition variation for the other three indices of 111,220 , and 400 , without overlapping by close index, were also examined using the same method. The diffractions from these three indices give the almost same results without any noticeable change from that of the Rietveld simulation. Hence, these results of three indices insist the random distribution on both $A$ - and $B$-sites.

This analysis of the peak intensity of the X-ray diffraction is still controversial. It should be noticed that the experimental results for the indices of 440 and 533 mean the higher angle diffraction with a small $d$ spacing of successive parallel atomic planes including $A$ - and $B$-sites. On the other hand, the 400 diffraction comes from the plane which consists of only atoms on $A$-site in the spinel structure. Unfortunately, we cannot conclude definitely at the present. The situation is less clear, but the results of Fig. 13 may provide a clue to understand the formation of the anomaly.

\subsection{Discussion of the hump anomaly}

The hump anomaly in the magnetization, as seen in Figs. 9-11, can be seen only a restricted composition region of $0.50 \leq x \leq 0.55$. The origin and the temperature variation of the anomaly have not been explained, so far. The characteristic features of the data are as follows: (a) The irreversibility of the hump appears in $M$ between the $\mathrm{ZFC}$ and the $\mathrm{FC}$ processes even above $T_{\mathrm{g}} \simeq 16 \mathrm{~K}$. Their bifurcation from the $\mathrm{ZFC}$ and $\mathrm{FC}$ arises around at 130 


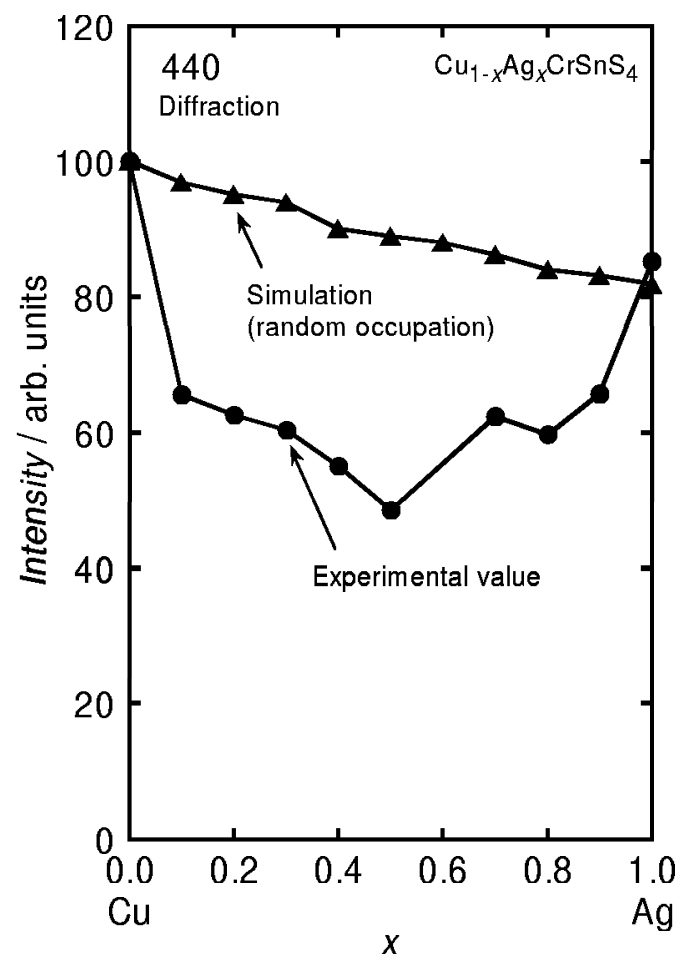

Figure 13: Composition dependence of the peak height (peak intensity) of the X-ray powder diffraction from the 440 planes of the Miller index for $\mathrm{Cu}_{1-x} \mathrm{Ag}_{x} \mathrm{CrSnS}_{4}$. The variation of the peak height is normalized by means of the magnitude for $\mathrm{CuCrSnS}_{4}$ with $x=0.0$ as 100 percent. Calculated peak height is also indicated, where this simulation is assumed to be random distributions on both of $A$ - and $B$-sites. 
$\mathrm{K}$ with decreasing temperature. The discrimination between the ZFC and the $\mathrm{FC}$ disappears around $1.0 \mathrm{kOe}$. In higher field than $1.0 \mathrm{kOe}$ the hump shrinks, exhibiting a tiny trace which is not shown in Fig. 10. (b) The hump of $M$ grows between 30 and $130 \mathrm{~K}$, and the local maximum $T_{\max }$ emerges at approximately $70-80 \mathrm{~K}$. The temperature $T_{\max }$ gives almost same values below 1000 Oe, as seen in Figs. 9 and 10. (c) The temperature $130 \mathrm{~K}$ is much larger than Weiss temperature of $-23.2 \mathrm{~K}$ as shown in Table 1 , therefore the anomaly indicates the absence of any magnetic long-range order.

The following interpretation on the anomaly is less clear, without a definite conclusion at present because of the absence of microscopic measurements. These experimental results would be attributed to the effects of a superimposition and/or coexistence of the spin-glass and cluster-glass formations based on the detection for the bifurcation from the ZFC and $\mathrm{FC}$ magnetization. Two glass-like phenomena take place in the different temperature regions. The long-range spin-glass freezing occurs below $T_{\mathrm{g}}=16 \mathrm{~K}$, whereas the cluster-glass appears dominantly between 30 and $130 \mathrm{~K}$ accompanied with the upturn hump anomaly in $M$.

With decreasing temperature, the germination of the clusters might takes place at $130 \mathrm{~K}$, and the blocking develops simultaneously at the same temperature, where the largest size cluster may contributes to the initial blocking. The bifurcation between the ZFC and FC magnetizations implies the blocking of the spin-clusters. Inside of a spin-cluster the interaction is stronger than that of environment, then, the resultant little large moments survive. The temperature variation of the anomaly might come from the combined effects via exchange, dipolar and Zeeman interaction in the disordered system introduced by dilution in the spin assembly, and by the local lattice distortion. These spin-clusters are embedded in the matrix of spin-glass elements where this matrix is already melted into paramagnetic phase $T>30$ $\mathrm{K}$. These spin-clusters float like islands in the background sea which consists of individual spin matrix. With further decreasing temperature, individual spins of the matrix are still active as paramagnetic state against temperature. Eventually, the long-range freezing has been fulfilled below $T_{\mathrm{g}}$. In the higher field, see Fig. 10, the magnetizations below $T_{\mathrm{g}}$ are quite similar to these of other concentration specimens. On the other hand, the low field magnetization indicates the splitting between the $\mathrm{ZFC}$ and $\mathrm{FC}$ curves even at $T_{\mathrm{g}}$ as can be seen in Fig. 9. Below $T_{\mathrm{g}}$, the spin clusters would survive and then the trace of the spin clusters can be detected in only lower field as seen in Fig 9. In higher fields, on the contrary, all the spins look like freezing 
withont the cluster.

In addition, we would like to point out here completely different view, even though this model is just a speculation at present. The potential two valence states of $\mathrm{Cu}$-ions are possible for $\mathrm{Cu}^{+}$or $\mathrm{Cu}^{2+}$ on the $A$-sites while $\mathrm{Cr}$ valence could change in part accordingly from $\mathrm{Cr}^{3+}$ to $\mathrm{Cr}^{2+}$ states on the $B$-sites. If this is the case, the $\mathrm{Cu}^{2+}$ has spin of $S=1 / 2$, consequently, the $A$ and $B$-sites have magnetic moments and the interaction between the nearest neighbor $A$ - and $B$-sites becomes larger than that in the only $B$-site situation. The connection and the interaction path is not known. Presumably, magnetic dimmer, triangle, and or linear chain through the $A$ - and $B$-sites can be constructed. These magnetic clusters may relate to 440 planes anomaly in Fig. 13 mentioned in the XRD. This valence change may be induced by temperature variation, even though we have no convincing evidence. In the valence fluctuation, the spin-clusters can be nucleated when the specimen is cooled, having correlations among them. The valence fluctuation may arise from the specific composition with $x=0.50$ on the $A$-site because the difference of ionic radius is fairly large between $\mathrm{Cu}^{+}$and $\mathrm{Ag}^{+}$ions.

In the end, the negative value of the magnetization in $10 \mathrm{Oe}$, as seen in the $\mathrm{ZFC}$ process of Fig. 12, remains strange. The net magnetization of the whole clusters must be anti-parallel to the external field. In the frozen clusters below $120 \mathrm{~K}$ in the ZFC process, the orientation of the magnetic moments of the clusters is not random accidentally but a little bias in the spatial distribution as shown in the specimen of Fig. 12. We stress that the negative value of the magnetization could not experimental error because the magnitude of the $\mathrm{FC}$ magnetization in 10 Oe recovers sufficiently and even larger than that of positive value in ZFC of Fig. 9, compare the results Fig. 9 and Fig. 12.

\subsection{A-T line of spin-glass}

The magnetic field dependence, $T_{\mathrm{g}}(H)$, has been studied theoretically by de Almeida and Thouless ( $A-T$ ) for the Ising model (27). This line is called A-T line. Experimentally, a typical example of the field dependence of the spin-glass transition $T_{\mathrm{g}}(H)$ has been reported by $\mathrm{A}$-Katori and Ito for the Ising spin-glass system of $\mathrm{Fe}_{x} \mathrm{Mn}_{1-x} \mathrm{TiO}_{3}$ (28).

An attempt has been made to examine the field dependence of the the spin-glass transition, $T_{\mathrm{g}}(H)$ for the present specimen with $x=0.50$. Table 2 lists the numerical value of the field dependence of the spin-glass transition temperature $T_{\mathrm{g}}(H)$ for $x=0.50$. We define, here, that $T_{\mathrm{g}}(H)$ is the spin-glass 


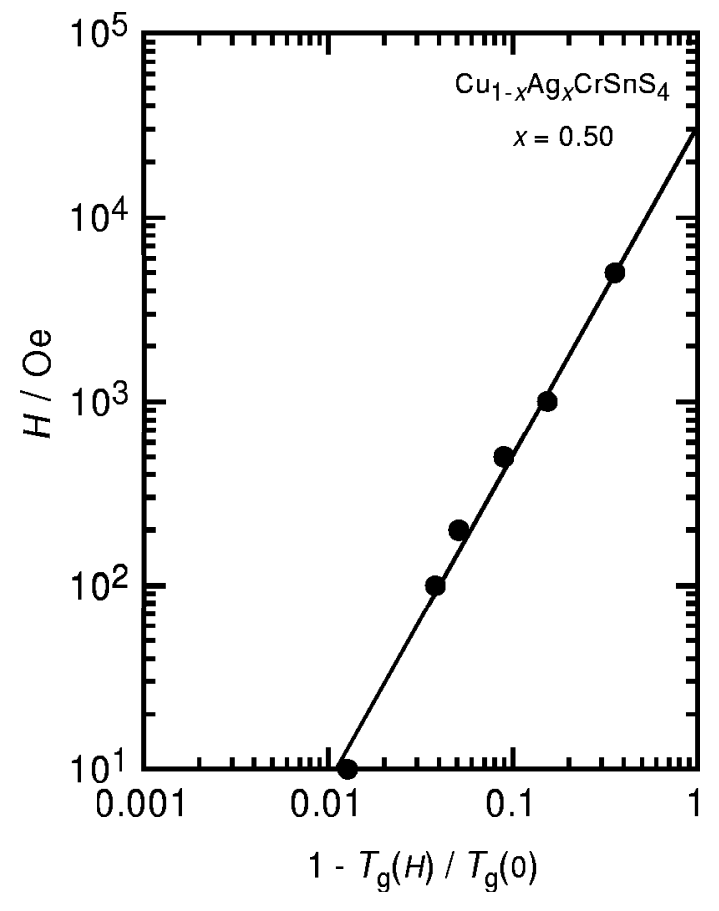

Figure 14: Magnetic field dependence of the spin-glass transition versus reduced temperature $1-T_{\mathrm{g}}(H) / T_{\mathrm{g}}(0)$ for $\mathrm{Cu}_{0.50} \mathrm{Ag}_{0.50} \mathrm{CrSnS}_{4}$. A formula of $H=A\left[1-T_{\mathrm{g}}(H) / T_{\mathrm{g}}(0)\right]^{\alpha}$ is used. The phase boundary between the paramagnetic and spin-glass phases is called A-T line. The value of $\alpha$ is obtained to be 1.77 . 
onset temperature of the appearance of irreversibility from the ZFC and $\mathrm{FC}$ magnetization. The temperature at the top for the cusp is taken as the $T_{\mathrm{g}}(H)$. The $T_{\mathrm{g}}(0)$ is, here, assumed to be $15.8 \mathrm{~K}$ for the value of 5.0 Oe at the lowest field of our measurements.

Fig. 14 shows the field dependence, $T_{\mathrm{g}}(H)$, for $x=0.50$. The value of $\alpha$ is obtained from the fitting of the formula of $H=A\left[1-T_{\mathrm{g}}(H) / T_{\mathrm{g}}(0)\right]^{\alpha}$ to the experimental results, where $A$ is a constant. The value of the exponent $\alpha$ is obtained to be 1.77 in the present specimen. The theory predicts $\alpha$ to be $3 / 2$ for the Ising system (27). Our spin system is not the Ising, but the Heisenberg model(12). The necessity to have $\alpha=3 / 2$ is not required for the Heisenberg system. Actually other Heisenberg system, for an example, $A g \mathrm{Mn}$ spin-glass, does not have $\alpha=3 / 2$ either (29).

\subsection{Isothermal $M-H$ curves for $x=0.50$}

Fig. 15 shows $M-H$ curves for $x=0.50$ at several temperatures up to $H$ $=10.000 \mathrm{kOe}$. Fig. 16 indicates expanded plots in low field regions up to 1000 Oe. Coercive forces $H_{c}$ for forward and backward direction are as follows: $\pm 350 \mathrm{Oe}$ at $5.0 \mathrm{~K}, \pm 20 \mathrm{Oe}$ at $20 \mathrm{~K}$, and $\pm 70 \mathrm{Oe}$ at $70 \mathrm{~K}$, respectively. No detection of $H_{\mathrm{c}}$ is obtained at $150 \mathrm{~K}$ because of the paramagnetic phase. The magnitude of $H_{c}$ at $70 \mathrm{~K}$ is larger than that at $20 \mathrm{~K}$, which might relate subtly to the existence of the local maximum in the hump anomaly of $M$ around $70 \mathrm{~K}$. The largest difference between the ZFC and $\mathrm{FC}$ process is detected at around $70 \mathrm{~K}$ in the hump anomaly in $M$. The origin of the hysteresis loop arises from the influence of the random field. If the system should become ferromagnetic with the multi-domain structure, the cause of loop would be completely different from the present system. Since the temperature region $T>T_{\mathrm{g}}$, the long-range freezing is destroyed, however, the short-range order exists owing to the existence of cluster-glass, then we expect more complicated behavior in the $M-H$ curves than that of the simple paramagnetic phase.

Next step we examine numerical magnitude itself for the magnetization in the $M-H$ curves. A degree of randomness for the orientation of spins will be verified in the spin-glass phase. One Cr atom exists in the formula unit for any value of $x$. The experimental value of magnetization $M$ is obtained to be 255 emu mol-f.u. ${ }^{-1}$ at $H=10.0 \mathrm{kOe}$ at $5.0 \mathrm{~K}$. This magnitude leads to the magnetic moment $n_{\mathrm{B}} \mathrm{Cr}$-atom ${ }^{-1}$ at $5 \mathrm{~K}$ in dimensionless numerical value. Here $n_{\mathrm{B}} \mathrm{Cr}_{\text {-atom }}{ }^{-1}=g S=M /\left(N_{\mathrm{A}} \times \mu_{\mathrm{B}}\right)=4.58 \times 10^{-2} \mathrm{Cr}$-atom ${ }^{-1}$, where $M$ is the moment in emu per mol-f.u., and $N_{\mathrm{A}}$ is the Avogadro's number. 


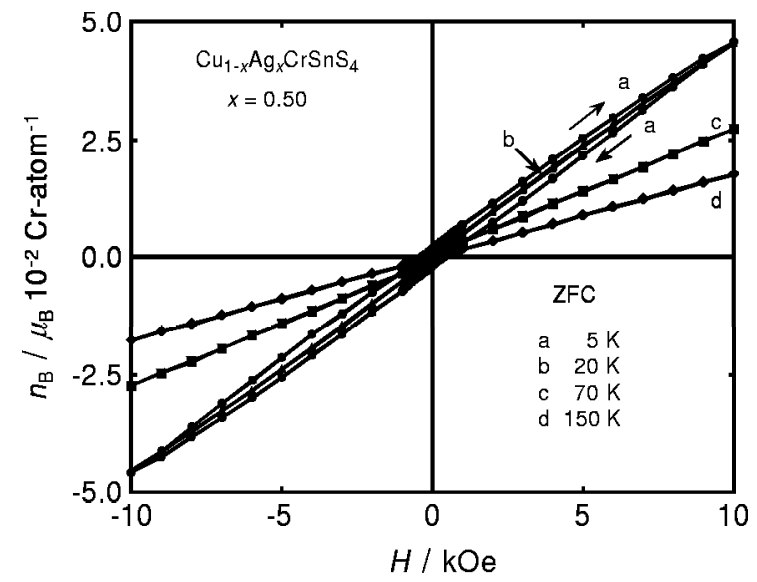

Figure 15: Magnetization as a function of applied magnetic field up to $10.0 \mathrm{kOe}$ for $\mathrm{Cu}_{0.50} \mathrm{Ag}_{0.50} \mathrm{CrSnS}_{4}$ with $x=0.50$ at several temperatures in the ZFC process. 


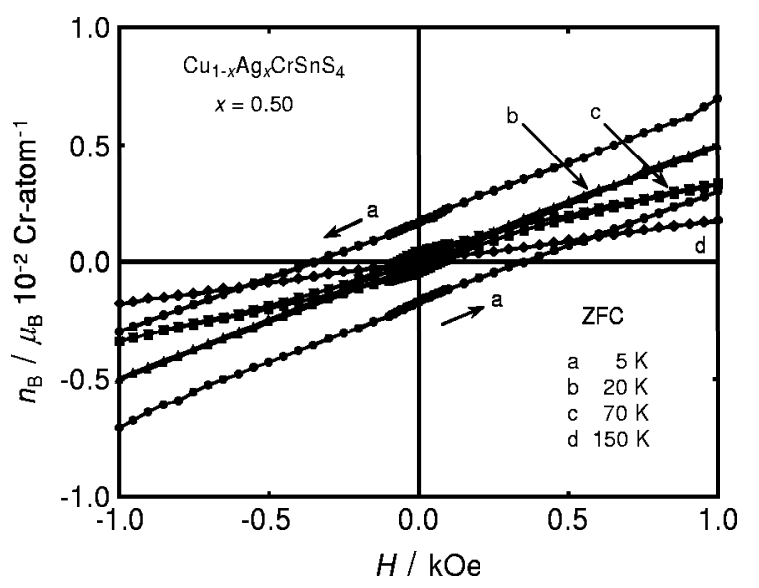

Figure 16: An enlargement of data of Fig. 15 in the field up to 1000 Oe in the ZFC process. Coercive forces $H_{\mathrm{c}}$ for forward and backward direction are as follows: \pm 350 Oe at $5.0 \mathrm{~K}, \pm 20$ Oe at $20 \mathrm{~K}$, and \pm 70 Oe at $70 \mathrm{~K}$. The value of $H_{\mathrm{c}}$ is higher at $70 \mathrm{~K}$ than that at $20 \mathrm{~K}$, reflecting the cluster-glass formation. 
On the other hand, the Curie-Weiss law supports strongly that the Cr atoms have a $\mathrm{Cr}^{3+}$ valence state, then, the saturated moment is expected to be $n_{\mathrm{B}}=3.00 \mathrm{f.u.}^{-1}=3.00 \mathrm{Cr}^{-a_{0}}{ }^{-1}$. The experimental values of the magnetization at $10.0 \mathrm{kOe}$ for $x=0.50$ are listed in Table 3 . The last column indicates the percent of the experimental results of $n_{\mathrm{B}} \mathrm{Cr}$-atom ${ }^{-1}$ on the saturated value of $n_{\mathrm{B}}=3.00$. The magnitude of $n_{\mathrm{B}} \mathrm{Cr}$-atom ${ }^{-1}$ is no more than $1.53 \%$ on the value of $S=3 / 2$ at $5.0 \mathrm{~K}$. These results demonstrate that the magnetic moments are frozen randomly in the spin-glass phase below $T_{\mathrm{g}} \simeq 16 \mathrm{~K}$. The spin-glass phase seems to be well established with random spin orientation. The low external field is insufficient to reach the value of saturation magnetization. It is noted that the spin-clusters themselves do not influence strongly on the $M-H$ curves of Figs. (15)-(16).

The hysteresis loops in a FC-process was measured in order to inspect the coercive force for increasing and decreasing external fields. Fig. 17 shows $M-H$ curve for $x=0.50$ at $5.0 \mathrm{~K}$ in a $\mathrm{FC}$-process. The specimen was cooled down to $T=5.0 \mathrm{~K}\left(T / T_{\mathrm{g}} \simeq 1 / 3\right)$ after field application of $1.0 \mathrm{kOe}$ at $300 \mathrm{~K}$. It was kept $10 \mathrm{~min}$., then the loop started with increasing field. Fig. 17 has the asymmetric value of the coercive force $H_{\mathrm{c}}$ due to the $\mathrm{FC}$ process. These values of the $H_{\mathrm{c}}$ are $H_{\mathrm{c}}=+250 \mathrm{Oe}$ and $-500 \mathrm{Oe}$, respectively. Fig. 18 shows a hysteresis loop for at $5.0 \mathrm{~K}$ in the $\mathrm{FC}$-process in $10.0 \mathrm{kOe}$. The measurement was begun from positive field of 10.0 to $-10.0 \mathrm{kOe}$ with decreasing field, because the maximum field of our apparatus was $10.0 \mathrm{kOe}$. Fig. 18 exhibits the magnitude of the $H_{\mathrm{c}}$, as $H_{\mathrm{c}}=+240$ Oe and -1150 Oe in $\mathrm{FC}$ of $10.0 \mathrm{kOe}$. The difference of the $H_{\mathrm{c}}$ in the asymmetry due to the bias field increases, presumably, continuously with the field in the $\mathrm{FC}$-process. The non-vanishing $H_{\mathrm{c}}$ means that the spin configuration in the frozen spinglass state due to the $\mathrm{FC}$-process is stable against the external field during hysteresis loop measurements.

\section{Summary}

A novel spin-freezing process of the cluster-glass in the matrix of spinglass elements has been proposed even above $T_{\mathrm{g}}$. The interpretation is based mainly on the clear observation of the irreversibility between $\mathrm{ZFC}$ and $\mathrm{FC}$ magnetization. The freezing of the spin-clusters does not show a long range order, but would be a glassy freezing, which is still compelling evidence and controversy. Only in a restricted composition region of $0.50 \leq x \leq 0.55$, an anomaly due to the cluster-glass formation can be realized. The dual 


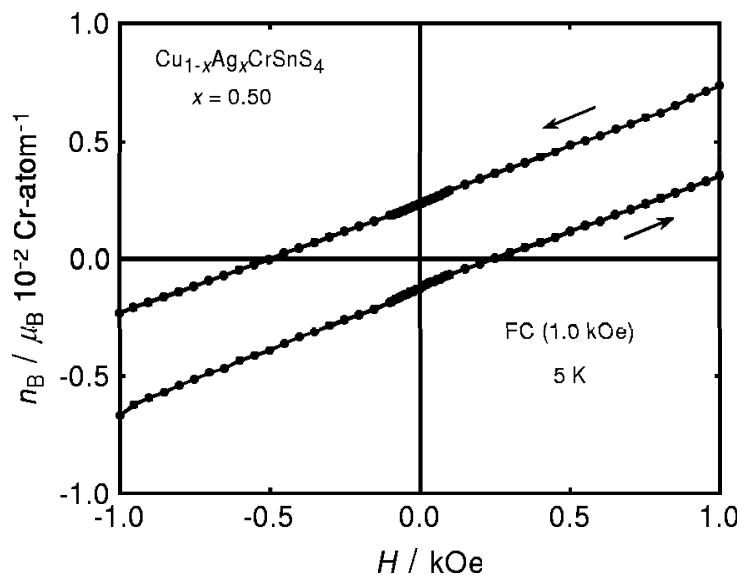

Figure 17: Field-cooled hysteresis loop at $5.0 \mathrm{~K}$. The field $1.0 \mathrm{kOe}$ had been applied at $300 \mathrm{~K}$ before cooling down. The specimen was kept $10 \mathrm{~min}$. at $5.0 \mathrm{~K}$, subsequently the measurement was begun. Arrows mark the direction of two branches (increasing or decreasing ) of the hysteresis loop. The coercive force $H_{\mathrm{c}}$ is asymmetric and they are $H_{\mathrm{c}}$ $=+250 \mathrm{Oe}$ and $H_{\mathrm{c}}=-500 \mathrm{Oe}$. 


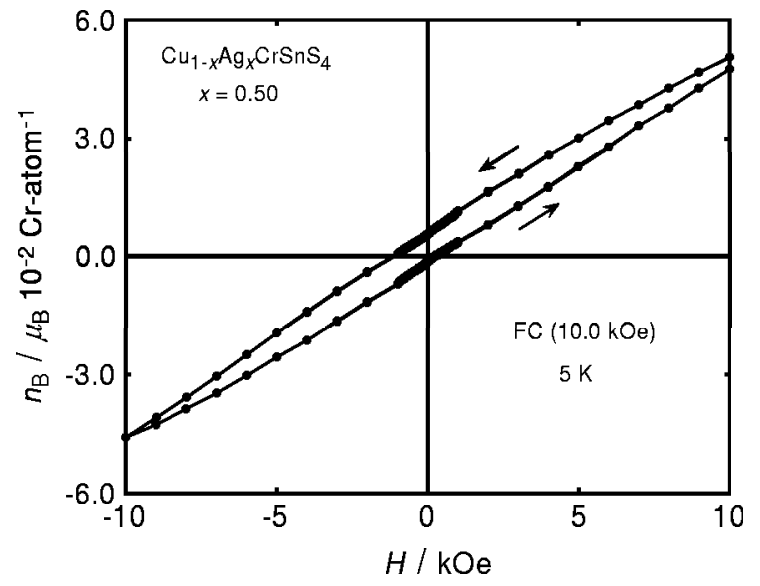

Figure 18: Field-cooled hysteresis loop at $5.0 \mathrm{~K}$. The field $10.0 \mathrm{kOe}$ had been applied at $300 \mathrm{~K}$ before cooling down. The coercive force $H_{c}$ are $H_{c}=+240 \mathrm{Oe}$ and $H_{\mathrm{c}}=-1150 \mathrm{Oe}$, respectively. 
non-magnetic substitutions on the $A$ - and $B$-sites are important and delicate in determining the cluster-glass formation, keeping resultant ferromagnetic interaction inside clusters. The measurements of dynamical properties of this system, and microscopic investigation are required to clarify the disordered system, as presented in $\mathrm{CuCrZrS}_{4}$ (30). It is our hope that the present study would lead to fruitful discussion and exotic physics relevant to random magnetic system.

\section{Acknowledgment}

The authors would like to thank Messrs. Fumihiro Kariya, Kenta Hasegawa, Yusuke Takikawa, Naoto Koseki, Hikaru Suzuki, and Kazuki Makino for their valuable experimental collaboration.

\section{References}

[1] F.K. Lotgering, in Proceedings of the International Conference on Magnetism, Nottingham (Institute of Physics and Physical Society, London, 1964), p. 533.

[2] F.K. Lotgering, Solid State Commun. 2 (1964) 55.

[3] H. Yokoyama, R. Watanabe, S. Chiba, J. Phys. Soc. Jpn 23 (1967) 450.

[4] R.P. van Stapele, F K. Lotgering, J. Phys. Chem. Solids 31 (1970) 1547.

[5] A. Kimura, J. Matsuno, J. Okabayashi, A. Fujimori, T. Shishidou, E. Kulatov, T. Kanomata, Phys. Rev. B 63 (2001) 224420.

[6] Y. Iijima, Y. Kamei, N. Kobayashi, J. Awaka, T. Iwasa, S. Ebisu, S. Chikazawa, S. Nagata, Philos. Mag. 83, (2003) 2521.

[7] T. Furubayashi, H. Suzuki, N. Kobayashi, S. Nagata, Solid State Commun. 131, (2004) 505.

[8] M. Ito, H. Yamamoto, S. Nagata, T. Suzuki, Physica B 383, (2006) 22.

[9] M. Ito, H. Yamamoto, S. Nagata, T. Suzuki, Phys. Rev. B 74, (2006) 214412. 
[10] Y. Fujimoto, T. Fujita, S. Mitsudo, T. Idehara, Y. Kawashima, S. Nagata, J. Magn. Magn. Mater. 310, (2007) 1991.

[11] H. Yamamoto, Y. Kawashima, K. Hondou, S. Ebisu, S. Nagata, J. Magn. Magn. Mater. 310, (2007) e-426.

[12] F. Kariya, S. Ebisu, S. Nagata, J. Solid State Chem. 182 (2009) 608 and references therein.

[13] F. Kariya, K. Ebina, K. Hasegawa, K. Koshimizu, B. Wuritunasitu, K. Hondou, S. Ebisu, S. Nagata, J. Solid State Chem. 182 (2009) 2018.

[14] H. Sekizawa, T. Okada, F. Ambe, in Proceedings of the International Conference on Magnetism, Moscow, ICM-73, 2(1973), p. 152.

[15] E. Riedel, W. Morlock, Z. Anorg. Allg. Chem. 438 (1978) 233.

[16] E. Riedel, W. Morlock, Z. Anorg. Allg. Chem. 474 (1981) 192.

[17] P. Colombet, M. Danot, G. Fillion, W.S. Glaunsinger, G. Calvarin, J. Magn. Magn. Mater. 51(1985) 265.

[18] I. Sarda, P. Colombet, G. Ablart, J. Pescia, C.R. Acad. Sci. Paris 308 Serie 2, (1989) 159.

[19] S.K. Misra, The American Physical Society 58 (1998) 22.

[20] V.A. Fedorov, Ya A. Kesler, E.G. Zhukov, Inorg. Mater. 39 Suppl 2, (2003) S68.

[21] Ya A. Kesler, E.G. Zhukov, D.S. Filimonov, E.S. Polulyak, T.K. Menshchikova, V.A. Fedorov, Inorg. Mater. 41 (2005) 914.

[22] G. Strick, G. Eulenberger, H. Hahn, Z. Anorg. Allg. Chem. 357 (1968) 338.

[23] G. Garg, S. Gupta, K.V. Ramanujachary, S.E. Lofland, A.K. Ganguli, J. Alloys Compd. 390 (2005) 46.

[24] K. Hasegawa, F. Kariya, K. Hondou, S. Ebisu, S. Nagata, in Proc. 25th Int. Conf. on Low Temperature Physics (LT25), Amsterdam J. of Physics: Conference Series 150 (2009) 042052. 
[25] F. Izumi, T. Ikeda, Mater. Sci. Forum 321-324 (2000) 198.

[26] S. Nagata, P. H. Keesom, and H. R. Harrison, Phys. Rev. B19 (1979) 1633.

[27] J.R.L. de Almeida and D.J. Thouless, J. Phys. A 11 (1978) 983.

[28] H.A. Katori and A. Ito, J. Phys. Soc. Japan 63 (1994) 3122, and references therein.

[29] R.V. Chamberlin, M. Hardiman, L.A. Turkevich, and R. Orbach, Phys. Rev. B 25 (1982) 6720.

[30] H.S. Suzuki, T. Furubayashi, Y. Kawashima, S. Nagata, T. Suzuki, T. Kawamata, I. Watanabe, T. Matsuzaki, A. Amato, Physica B 404 (2009) 649. 
Table 1.

Summary of the magnetic properties of $\left(\mathrm{Cu}_{1-x} \mathrm{Ag}_{x}\right) \mathrm{CrSnS}_{4}$ based on the Curie-Weiss law. The value of $p_{\text {eff }} \mathrm{Cr}^{-a t o m}{ }^{-1}$ is extracted from an assumption that only $\mathrm{Cr}$ atoms possess a localized magnetic moment.

\begin{tabular}{cccccc}
\hline$x$ & $T_{\mathrm{g}}$ & $C$ & $\theta$ & $p_{\text {eff }}$ & $\begin{array}{c}\text { Tempertature range } \\
\text { over the C-W law }\end{array}$ \\
Composition & $(\mathrm{K})$ & $\left(\right.$ emu K mol-f.u. $\left.{ }^{-1}\right)$ & $(\mathrm{K})$ & $\left(\right.$ Cr-atom $\left.^{-1}\right)$ & $(\mathrm{K})$ \\
\hline 0.00 & 18.0 & 1.67 & -2.86 & 3.66 & $200-350$ \\
0.20 & 17.4 & 1.82 & -4.67 & 3.82 & $200-350$ \\
0.40 & 15.6 & 1.90 & -26.3 & 3.90 & $200-350$ \\
0.50 & 15.2 & 1.81 & -23.2 & 3.81 & $230-350$ \\
0.60 & 15.8 & 1.98 & -22.5 & 3.97 & $200-350$ \\
0.70 & 16.2 & 1.89 & -23.6 & 3.89 & $200-350$ \\
1.00 & 18.0 & 1.92 & -0.90 & 3.92 & $220-350$ \\
\hline
\end{tabular}


Table 2.

The numerical value of field dependence of the spin-glass transition temperature $T_{\mathrm{g}}(\mathrm{K})$ for $\mathrm{Cu}_{0.50} \mathrm{Ag}_{0.50} \mathrm{CrSnS}_{4}$.

\begin{tabular}{ccc}
\hline$H(\mathrm{Oe})$ & $T_{\mathrm{g}}(\mathrm{K})$ & $1-T_{\mathrm{g}}(H) / T_{\mathrm{g}}(0)$ \\
\hline 5 & 15.8 & \\
\hline 10 & 15.6 & 0.013 \\
\hline 100 & 15.2 & 0.038 \\
\hline 200 & 15.0 & 0.051 \\
\hline 500 & 14.4 & 0.089 \\
\hline 1000 & 13.4 & 0.152 \\
\hline 5000 & 10.2 & 0.354 \\
\hline
\end{tabular}


Table 3.

The numerical value of magnetic moment $n_{\mathrm{B}}=g S=2 S$ for $x=0.50$ of $\mathrm{Cu}_{0.50} \mathrm{Ag}_{0.50} \mathrm{CrSnS}_{4}$, where $g$ is the Lande's $g$-factor. These results are obtained from the values at $H=10.0 \mathrm{kOe}$ at several temperatures for the ZFC-process in Fig. 14. The last column indicates the percent of $n_{\mathrm{B}}$ Cr-atom ${ }^{-1}$ on $n_{\mathrm{B}}=3.00$ for the saturated value of $\mathrm{Cr}^{3+}$.

\begin{tabular}{cccc}
\hline$T$ & $M$ & $n_{\mathrm{B}}$ & percent \\
\hline$(\mathrm{K})$ & $\left(\right.$ emu mol-f.u. $\left.{ }^{-1}\right)$ & $\left(\mu_{\mathrm{B}}\right.$ Cr-atom & \\
\hline 5 & $2.55 \times 10^{2}$ & $4.58 \times 10^{-2}$ & $\%$ \\
20 & $2.53 \times 10^{2}$ & $4.55 \times 10^{-2}$ & 1.53 \\
70 & $1.52 \times 10^{2}$ & $2.73 \times 10^{-2}$ & 1.52 \\
150 & $9.87 \times 10$ & $1.77 \times 10^{-2}$ & 0.910 \\
\hline
\end{tabular}

